OS11 (P) - 29 (OS11W0023)

\title{
Effects of Surface Treatment on Fatigue Properties of an lon-Nitriding Low Carbon Steel
}

\author{
Takashi Makishi, Dept. of Mechanical Systems Engineering, Univ. of the Ryukyus, Nakagami-gun, Japan \\ Chobin Makabe, Dept. of Mechanical Systems Engineering, Univ. of the Ryukyus, Nakagami-gun, Japan \\ Hideo Kaneshiro, Dept. of Mechanical Systems Engineering, Univ. of the Ryukyus, Nakagami-gun, Japan
}

The fatigue strength and the fracture mechanisms of ion-nitrided steel were investigated using four types of specimens - annealed specimens, nitrided specimens, specimens from which the surface layer was removed (surface-removed specimens), and multi surface treated specimens with ion-nitriding and shot peening. In the case of surface-removed specimens, the surface layers were removed after nitriding, reducing their radius by $0.1 \mathrm{~mm}$. The shot peening was carried out after or before the nitriding, because the treatment's order of nitriding and shot peening did not affect on the fatigue property. The fatigue limit of the nitrided specimens was higher than that of the annealed specimens, but was almost the same as that of the surface-removed specimens. This is related to the hardness distribution of the specimens. In the case of the nitrided specimen, the maximum hardness was recorded at the surface and decreased gradually toward the center of the specimen. The estimated strength deduced from the hardness of that specimen is higher than the measured fatigue limit within a depth of $0.1 \mathrm{~mm}$ from the surface. At internal depths of more than $0.1 \mathrm{~mm}$ from the surface, the estimated strength is expected to be lower than the fatigue limit. Accordingly, the fatigue limit and strength of the nitrided specimens are determined by the internal properties of the materials, and thus the fatigue limit and strength of the nitrided specimens are not decreased even if the surface layer is removed. In the case of the nitrided specimens, the cracks were difficult to be observed from the surface because the crack initiations occurred internally. Since removing the surface layers facilitates observing the cracks on the surface, the initiation mechanism of internal crack can be evaluated by observing the surface crack initiation. From the observation of the fracture surfaces, a similar mechanism is assumed to be operating internally in the nitrided specimens. Fatigue strength of nitrided specimen could be more improved by the shot peeng. From those results, it is found that the fatigue strength of the nitrided low carbon steel was related to the hardness distributions. 